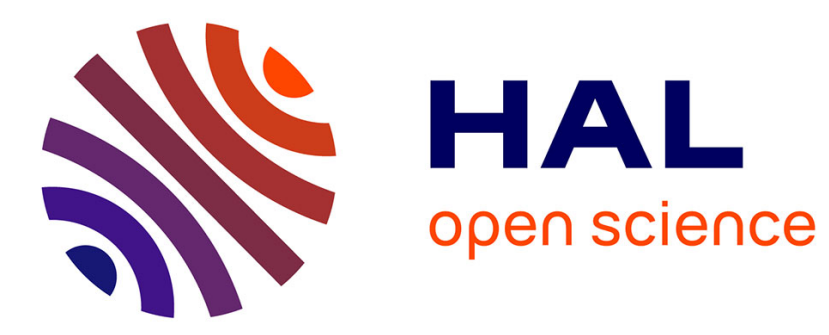

\title{
Long-time damage under creep experiments in disordered materials: Transition from exponential to logarithmic fracture dynamics
}

\author{
Claudio Fusco, L. Vanel, D. R. Long
}

\section{To cite this version:}

Claudio Fusco, L. Vanel, D. R. Long. Long-time damage under creep experiments in disordered materials: Transition from exponential to logarithmic fracture dynamics. European Physical Journal E: Soft matter and biological physics, 2013, 36 (4), pp.34. 10.1140/epje/i2013-13034-y . hal-01727361

\section{HAL Id: hal-01727361 \\ https://univ-lyon1.hal.science/hal-01727361}

Submitted on 12 Mar 2018

HAL is a multi-disciplinary open access archive for the deposit and dissemination of scientific research documents, whether they are published or not. The documents may come from teaching and research institutions in France or abroad, or from public or private research centers.
L'archive ouverte pluridisciplinaire HAL, est destinée au dépôt et à la diffusion de documents scientifiques de niveau recherche, publiés ou non, émanant des établissements d'enseignement et de recherche français ou étrangers, des laboratoires publics ou privés. 


\title{
Long-time damage under creep experiments in disordered materials: Transition from exponential to logarithmic fracture dynamics
}

\author{
C. Fusco ${ }^{1}$, L. Vanel ${ }^{1,2}$, and D. R. Long ${ }^{1}$ \\ 1 Laboratoire de Polymères et Matériaux Avancés, CNRS/Rhodia, 85 avenue des Frères Perret, F-69192 Saint Fons Cedex, \\ France \\ 2 Laboratoire de Physique de la Matière Condensée et Nanostructures, Université de Lyon, Université Lyon 1 and CNRS, UMR \\ 5586, F-69622 Villeurbanne Cedex, France
}

the date of receipt and acceptance should be inserted later

\begin{abstract}
Some materials, and in particular some polymer materials, can display an important range of stress levels for which slow and progressive damage can be observed before they finally break. In creep or fatigue experiments, final rupture can happen after very long times, during which the mechanical properties have progressively decayed. We model here some generic features of the long-time damage evolution of disordered elastic materials under constant load, characterized by a progressive decrease of the elastic modulus. We do it by studying a two-dimensional electric random fuse network with quenched disorder and thermal noise. The time evolution of global quantities (conductivity or, equivalently, elastic modulus) is characterized by different regimes ranging from faster than exponential to slower than logarithmic, which are governed by the stress level and the relative magnitude of disorder with respect to temperature. A region of widely distributed rupture time exists where the modulus decays (more slowly than) logarithmically for not too small values of the disorder and for not too large values of the load. A detailed analysis of the dynamical regimes is performed and presented through a phase diagram.
\end{abstract}

\section{Introduction}

Materials, and in particular polymer materials, can be brittle or ductile. When submitted to an increasing stress, they can either break suddenly above a critical value, or undergo progressive damage as stress increases up to a stress level where final rupture of the sample occurs. An important parameter in the appearance of progressive damage is the disordered nature of materials [1-3]. When submitted to a constant load, materials break due to thermally activated processes which produce a slow rupture dynamics (see [4] and references therein). Depending on the nature of the material and the constant load level, the amount of damage accumulated at the time of catastrophic rupture can be almost negligible or instead very large. While many materials undergo very little damage before final rupture, polymer materials can display an important range of stress levels over which slow and significant progressive damage will be observed, contributing to creep until the final rupture. Being able to control the evolution of the mechanical properties during the slow damage regime as well as the duration of this regime is crucial for applications in which the finite life-time of materials is an important issue.

Correspondence to: loic.vanel@univ-lyon1.fr
For instance, glass fiber reinforced polyamides, used in the automotive industry thanks to their light weight in comparison to metals for the same rigidity, are submitted to a relatively high level of stress, during a long time. Standard cyclic fatigue tests [5] are usually performed in order to determine stress versus lifetime curves for these polymer composite materials, typically at frequencies of a few $\mathrm{Hz}$ or a few tens of $\mathrm{Hz}$ and at different temperatures $[7,6]$. More generally, the mechanical behavior of semi-crystalline polymers has been widely studied over the past decades and is still an intense research subject $[8-13,16,17,14,15,18]$. At small stresses the elastic and viscoelastic behavior of semi-crystalline polymer involves the interlamellar amorphous phase, and at higher stresses the plastic behavior involves the crystalline phase. The mechanical properties of the polymers drops as a function of the duration during a fatigue test, even if the maximum stress belongs to the elastic or viscoelastic domain. A part of irreversible damage occurs and this damage is not completely understood. Kuksenko and Tamusz have studied polymers in situ by SAXS [19] and observed the formation of microvoids during a tensile experiment. The size of the microvoids remain constant and the breaking occurs when a critical density of microvoids is reached. In oriented polymers the size of the cavities is of a few tens of nanometers, whereas in non oriented polymers the 
size of the cavities is about some thousands of nanometers. Recently, Castagnet et al [14] characterized the polyvinylidene fluoride (PVDF) by volumetric measurements during tensile test and by SAXS. It has been shown that cavities occurs in the interlamellar amorphous phase just before yielding. Pawlak and Galeski [15] studied the cavitation and the crystal shearing. In polyamide, because of the high resistance of the crystals, the main damage mechanism is cavitation.

The microscopic origin of the damage is still a subject of scientific studies, and depend on the material. However, we argue here that some generic features should be present in a very wide class of materials for which time plays an essential role in the damage mechanisms. In such circumstances, we assume that damage is related to nucleation of defects, or cavities, which grow in number, or which may grow in size. The dominant damage mechanism results therefore from the competition between the two following mechanisms: either 1) the increase of the number of cavities thanks to independent nucleation processes throughout the sample, until the number of cavities reaches a critical value above which the material crumbles, or 2) the growth of a single defect which results in the apparition of a fracture in the material.

In this manuscript, we assume that the nucleation of defects is an activated process which takes place on a time scale much longer than any microscopic time scales $\left(10^{-12} s\right.$ on the molecular scale). The barriers depend on the applied stress, and for the slow rupture process that we are interested in, the corresponding barriers for nucleation are larger than a few tens of $k_{B} T$ so that each nucleation event takes place on a macroscopic time scale. As we shall see, materials for which a slow and progressive damage regime do exist, on time scale which may be longer than a week or more, are characterized by a wide distribution of nucleation energy barriers. The transition between mechanisms 1) and 2) may be obtained when reducing the width of the energy barrier distribution, so that the increase of stress ahead of a small defect may be sufficient to induce on time scale short enough the nucleation of another cavity just ahead of this defect, resulting thus in a catastrophic fracture process.

The aim of this manuscript is to show how one can determine the phase diagram corresponding to the various damage behaviors on a wide range of time scales, as a function of the width of the energy barriers distribution, the temperature, and the stress level. We do not specify the nature of the microscopic damage, but we just assume that it appears through a nucleation mechanism where the distribution of nucleation barriers characterizes a disorder in the rupture properties of the material. Once a microscopic damage has nucleated the broken region can no longer bear stress and the latter is redistributed assuming elastic interactions. The competition between disorder and elasticity is crucial, because it can be responsible for long-time damage and fatigue of the sample and can determine a transition from brittle to ductile behaviour when the disorder becomes dominant. Indeed, many theoretical studies have shown that depending on the strength of the disorder and stress level different regimes may appear, such as abrupt rupture, rupture with localized damage or rupture with diffuse damage $[1-3,20-26]$.

Three dimensional elastic behavior in a disordered materials is quite costly to calculate. Thus, we chose to study a simpler model, where the $3 \mathrm{D}$ elastic displacement is replaced by a scalar quantity analogous to an electrical potential and the elastic stress is replaced by the electrical intensity in a 2D electrical network. Computation is therefore less demanding and allow to reach longer time scales which are the central purpose of our study. Such models, so-called random fuse network (RFN) or fibre bundle models (see [3] and refs. therein), have two interesting limiting cases which correspond to the two damage mechanisms we would like to model: weak disorder, where cracks quickly nucleate and propagate up to macroscopic fracture, and strong disorder, where more extensive damage develops before rupture $[1,20,31,37]$.

Up to now, thermally activated rupture damage in RFN models has been studied mainly in 1D. It was shown that overall disorder will decrease the lifetime of the network [32-36] as long as less than $50 \%$ of the network is broken [36]. However, it was also found that the rate of broken fuses is non monotonous in time: first it decreases mostly as $1 / t$ (thus resulting in a logarithmic decay of the damage) and then it increases until macroscopic rupture occurs [35]. In comparison, the full dynamics of the 2D RFN with disorder and thermal noise is little explored [37, 38]. A clear connection between the actual dynamical response of the 2D RFN under constant applied stress and the damage dynamics as a function of disorder, temperature and external loading is still lacking. In fact, the 2D dynamics turns out to be richer and much more complex than in 1D because of the competition between disorder and stress intensification due to stress concentration at microcrack tips, which results in a non-uniform redistribution of stress and in possible cascade ruptures. These ingredients make the $2 \mathrm{D}$ RFN more realistic and closer to experimental systems, and thus more useful to understand the fracture mechanics and the fatigue mechanisms of disordered materials.

In this paper, we present a study of the rupture dynamics by means of a $2 \mathrm{D}$ RFN at constant imposed current in different regimes of temperature and disorder and analyze in detail the conditions under which a logarithmic decay of the fracture process can be observed. Our results suggest a transition from exponential to logarithmic dynamical evolution as disorder increases. The paper is organized as follows. In Sec. 2 we briefly recall the random fuse network. In Sec. 3 we discuss the results about the dynamical evolution of the modulus of the network and the occurrence of different dynamical regimes. A detailed analysis of these dynamical regimes is reported in Sec. 4 through a phase diagram, with the emphasis on the logarithmic regime associated to the slow damage dynamics of the network. The last section is devoted to some concluding remarks and perspectives. 


\section{Model}

We consider a 2D RFN of size $M \times N$ with square lattice topology at finite temperature, $M$ and $N$ being the number of nodes in the $x$ and $y$ direction respectively (see also Ref. [37]). Each of the bonds in the network is a fuse with unit electrical conductance. The network is driven by a constant current $i_{0}$ (constant force per spring $F_{0}$ in the spring system) applied at the top of the system, yielding a total current $M i_{0}$ flowing through the network. We notice that the $2 \mathrm{D} R \mathrm{RFN}$ is equivalent to a square lattice of springs in antiplane deformation. At variance with the 1D RFN, where the load is shared equally among the unbroken fuses, the elasticity in the 2D geometry allows for stress concentration at microcrak tips, leading to non-uniform stress redistribution and to a competition between aleatory rupture events and cascade effects. A scheme of the model showing this kind of effects is displayed in Fig. 1.

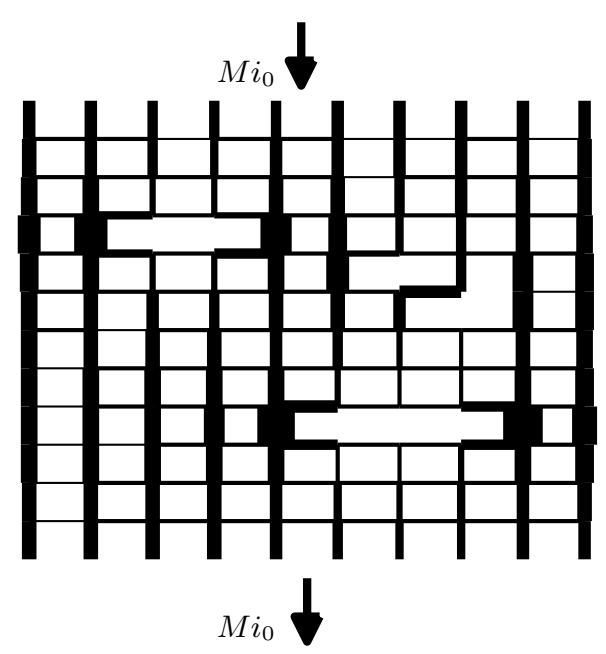

Fig. 1. Scheme of the $2 \mathrm{D} R F N$ on a square lattice. A total current $M i_{0}$ is applied at the top of the system in the vertical direction. The intact horizontal (vertical) fuses are represented by horizontal (vertical) lines whose thickness is proportional to the local current $i_{\ell}$. No line is drawn for the broken fuses. Notice that in the vicinity of broken fuses a higher local current flows on the intact vertical fuses.

Two important ingredients of the model are disorder and thermal effects. Each fuse $k$ carries a current rupture threshold $i_{c}(k)$. We introduce quenched randomness in the model by extracting the thresholds $i_{c}$ from a uniform probability distribution with mean $\left\langle i_{c}\right\rangle=1$ and variance $T_{d}$. This represents a disordered distributions of heterogeneities in the sample, as found for instance in polymer composites. In order to model the system at finite temperature we also add temporal thermal fluctuations in local currents $\delta i_{\ell}(k)$ on each fuse $k$, following a Gaussian distribution with zero mean and variance $T$. A fuse will break irreversibly when the total current across the fuse overcomes the current threshold on that fuse given by the distribution of disorder, as pictorially illustrated in Fig. 2. Thermal fluctuations add to the local current $i_{\ell}$ on a given fuse and can allow for rupture of a fuse even if $i_{\ell}<i_{c}$. In this model, the mean rupture time $\tau$ of a fuse can be

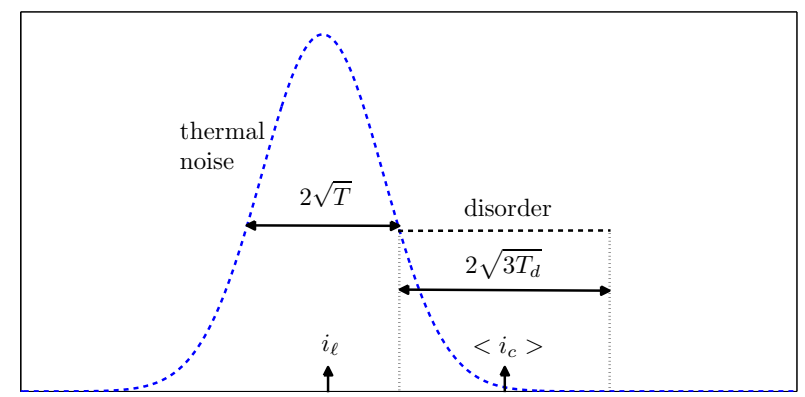

Fig. 2. Sketch of the probability distribution thermal fluctuations (left) and of rupture thresholds (right), pictorially illustrating the effect of thermal effects and disorder respectively. Current thresholds are distributed uniformly with a variance $T_{d}$ (corresponding to a width $2 \sqrt{3 T_{d}}$ of the distribution) around a mean value $\left\langle i_{c}\right\rangle$, while thermal fluctuations follow a Gaussian distribution with variance $T$ and are superimposed to the local current $i_{\ell}$.

expressed as

$$
\tau=\tau_{0} \exp \left[\frac{\left(i_{c}-i_{\ell}\right)^{2}}{2 T}\right],
$$

where $\tau_{0}$ is a time prefactor. The failure time $\tau_{F}$ (lifetime) of the entire network will occur when no current can flow anymore through the system. In the next sections we present the results of the numerical simulations of this model [40].

\section{Rupture dynamics at constant applied force (current)}

The dynamical behaviour of the system can be characterized by the global conductivity $E$ of the network, which is defined by the ratio of the total applied current $M i_{0}$ to the net potential difference between the top and bottom of the system, $\Delta V_{c c}$ :

$$
E=\frac{M i_{0}}{\Delta V_{c c}}
$$

Eq. (2) would correspond to the elastic modulus if we define the system in terms of forces and displacements, so that $E$ is the ratio between the applied force and the net displacement of the network.

In one dimension, $E$ is directly related to the fraction of broken fuses $\Phi(t)=n(t) / N_{t o t}$ through the relation

$$
E(t)=E_{0}(1-\Phi(t))
$$

where $n(t)$ is the number of broken fuses, $N_{t o t}$ is the total number of fuses and $E_{0}=M$ in our units. The initial 
rupture behavior is such that $n(t) \sim \ln t[35]$ so that $E(t)$ is expected to decrease logarithmically with time. In two dimensions, a good approximation of Eq. (3) for random damage is $E \simeq E_{0}(1-2 \Phi)[38,39]$. Thus, we also expect a simple relationship between the temporal evolution of the modulus and the damage as measured by the fraction of broken bonds.

In order to see the influence of the different parameters on the dynamical evolution of the $2 \mathrm{D}$ RFN we plot the modulus as a function of time in Fig. 3 by fixing two of the parameters $T_{d}, T$ and $i_{0}$ and letting the third one vary. The last point in time for each curve corresponds
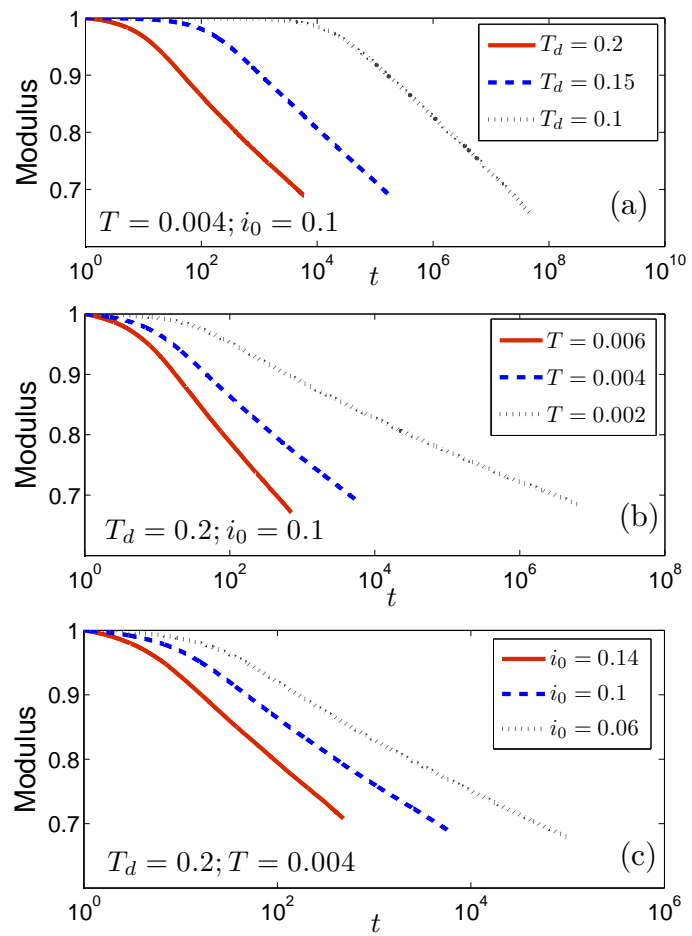

Fig. 3. Time evolution of the modulus for $T=0.004, i_{0}=0.1$ and three values of $T_{d}(\mathrm{a}), T_{d}=0.2, i_{0}=0.1$ and three values of $T$ (b), $T_{d}=0.2, T=0.004$ and three values of $i_{0}$ (c).

to the lifetime $\tau_{F}$ of the network for that given set of parameters: beyond this point catastrophic rupture occurs and the modulus abruptly becomes zero. In the $2 \mathrm{D}$ RFN we observe the same general qualitative trend with respect to the parameters $T_{d}, T$ and $i_{0}$ as in the $1 \mathrm{D}$ model: an increase in current, temperature or disorder reduces the lifetime of the network.

We find that the functional form of $E(t)$ crucially depends on the choice of $T_{d}, T$ and $i_{0}$. Some prototypical behaviours are illustrated in Fig. 4 , which shows the dynamical evolution of the modulus for $i_{0}=0.1$ and for four values of the disorder $\left(T_{d}=0.001,0.1,0.15,0.2\right)$. For very low $T_{d}$ the dynamical behaviour is dominated by thermal fluctuations and the modulus will decay mostly exponentially in time as it can be seen in Figs. 4(a)-(b). This exponetial behavior can be interpreted as follows. For the
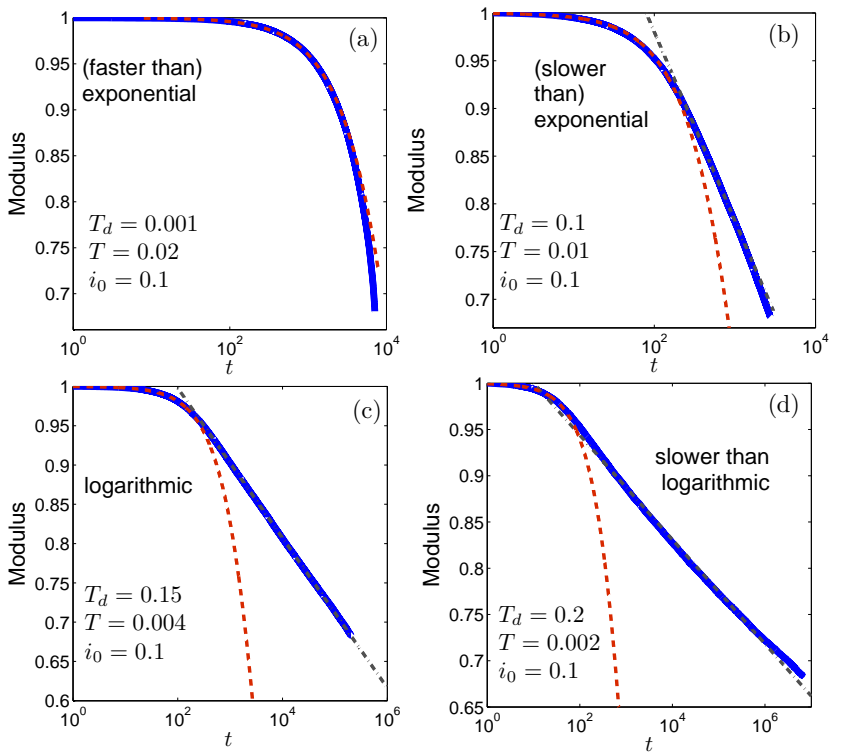

Fig. 4. Time evolution of the modulus (solid lines) for $i_{0}=0.1$ and for four different values of the disorder as indicated in the legends. The dashed lines in (a) and (b) are exponential fits to the data, while the dashed-dotted lines in (b)-(d) are logarithmic fits.

most part of the process, i.e. until almost the final rupture, each fuse breaks independently from each other, with a similar time scale, because the disorder (i.e. the barrier distribution) is narrow. Each fuse breaks following independent Poisson (exponential) processes as it is usual in independent nucleation processes. At some point, the intensification of stress is sufficient to induce fracture propagation corresponding to the final rupture of the sample. The conclusion is that, in this regime, the effect of stress redistribution can be considered negligible.

If we increase $T_{d}$ we see, after an initial exponential transient, a logarithmic decay of $E(t)$ (see Fig. 4(c)), which is characteristic of a slow damage dynamics of the network, as we will discuss in Sec. 4 . For larger values of $T_{d}$ the modulus develops a slower than logarithmic tail, as it is clear from Fig. 4(d). At the same time, we can observe that the value of the modulus just before the final rupture event depends rather weakly on $T_{d}$, especially at higher values of $T_{d}$, and is mainly determined by the value of $i_{0}$.

The transition from exponential to logarithmic rupture dynamics as a function of disorder is a novel aspect of the 2D RFN that has not been studied in detail so far. In fact, previous studies on the $1 \mathrm{D}$ RFN were mainly carried out in the strong disorder limit where it was found that the rate of broken fuses follows a $1 / t$ decay, the socalled Omori law, thus yielding a logarithmic evolution of the modulus $[35,36]$. In our model we can qualitatively explain the transition by considering the time distribution of individual rupture events, i. e. by looking at the times at which fuses break in the network. In the exponential regime these times are rather equally distributed, while in the logarithmic one they are rather scattered and very different. This is due to the fact that for low disorder there 
is not much difference in the rupture thresholds, so that rupture events can occur everywhere in the system with almost the same probability; on the other hand, for large disorder, rupture thresholds differ considerably and the rupture process break fuses with progressively increasing rupture thresholds [35]. The time scales associated to the weakest fuses are the shortest, thus the latter break first. The strongest fuses break on a longer time scales. Note that there is no causation in this regime, since stress redistribution is negligible here: each fuse break randomly with, statistically, its own time scale associated with local stress level and critical stress level.

By closely examining Fig. 4 one can clearly see that, irrespective of the value of $T_{d}$, there is always an initial transient of $E(t)$ that can be described by an exponential evolution. Moreover, the relative width of this transient regime (i.e. the width compared to the lifetime) decreases as a function of $T_{d}$. Even if the rupture times of individual fuses are broadly distributed, yielding a logarithmic evolution of the modulus, there is always an initial stage where independent rupture events with very similar rupture times occur, leading to an initial exponential decay of the modulus. The timescale of this initial exponential decay is set by the lowest rupture threshold (or lowest energy barriers) of the sample, here corresponding to $1-\sqrt{3 T_{d}}$.

\section{Analysis of the dynamical behavior}

The occurrence of the logarithmic regime has been investigated in detail through a proper data analysis the aim of which is to build a phase diagram where we can distinguish definite regions of the parameter space characterized by different dynamical evolutions of the modulus. We look for a form of the first derivative of the modulus of the type

$$
\frac{d E}{d t}=-\frac{E_{0}}{N} \frac{K}{t^{p}}
$$

where $K$ is a constant and $p$ is the analogous of the Omori exponent in $2 D$. The goal is to find the exponent $p$. If $p=1$ with a prescribed numerical precision, the evolution of $E$ will be logarithmic. If $p \neq 1$ there will be a deviation from the logarithmic evolution of $E$ (linear evolution in log-lin scale). If $p<1$, the evolution is faster than logarithmic and if $p>1$ the evolution is slower than logarithmic. We also compare the evolution of the modulus to a pure exponential decay. The details of the data analysis are explained in Appendix A.

The phase diagram obtained with this analysis is presented in Fig. 5 in the plane $\left(x \equiv \sqrt{T_{d}} / T, i_{0}\right)$, where three well-defined regions can be distinguished: $p>1$ (squares), $p<1$ and slower than exponential (circles), $p<1$ and faster than exponential (diamonds). Qualitatively we see that the slower than logarithmic regime $(p>1)$ occurs for rather high values of $x(x>70)$ and for small values of $i_{0}$ $\left(i_{0}<0.22\right)$.

In order to justify the choice of the parameter $x$ and to trace analytical curves separating the three regions we argue that the logarithmic and the exponential regimes correspond to given values of the width of the rupture time

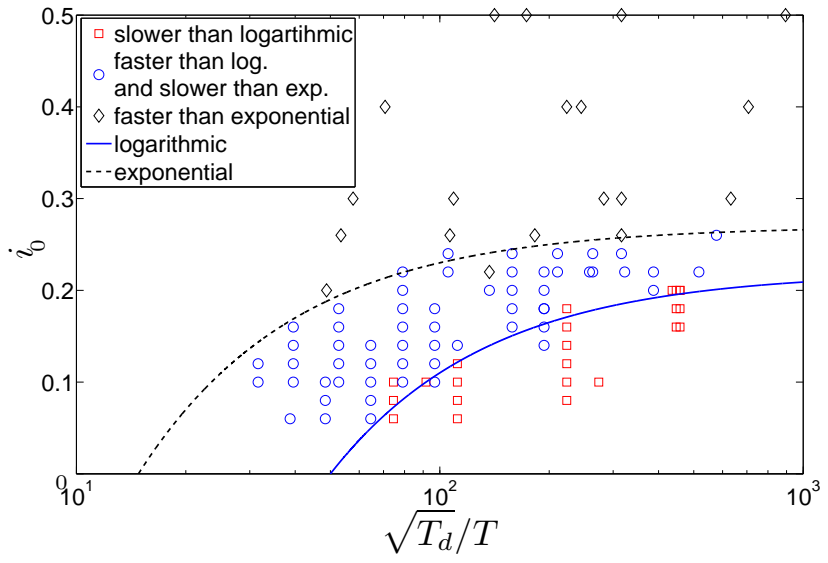

Fig. 5. Phase diagram in the $\left(x \equiv \sqrt{T_{d}} / T, i_{0}\right)$ plane: the open squares, the open circles and the open diamonds correspond to triplets $\left(T_{d}, T, i_{0}\right)$ for which the modulus follows a slower than logarithmic evolution, a faster than logarithmic (but slower than exponential) evolution and a faster than exponential evolution respectively. The two lines are curves of the form $A-C / x$ that have been plotted to visually distinguish the three regions. The solid (dashed) line should ideally correspond to points triplets for which the modulus displays a "truly" logarithmic (exponential) evolution. $A=0.22$ and $C=11$ for the solid line, $A=0.27$ and $C=4$ for the dashed line.

distribution of individual events. In fact, as explained at the end of Sec. 3, we expect that the rupture time distribution governs the occurrence of the different dynamical regimes, and specifically a rather narrow (large) time distribution for the exponential (logarithmic) regime. Let us consider first the rupture time distribution of individual fuses carrying a current $i_{\ell} \simeq i_{0}$ when the network is still intact. The ratio $\mathcal{R}$ between the maximum rupture time $\tau_{\max }$ and the minimum rupture time $\tau_{\min }$ in the network can be obtained from Eq. (1) where $i_{c}=\left\langle i_{c}\right\rangle \pm \sqrt{3 T_{d}}$ :

$$
\mathcal{R}=\frac{\tau_{\max }}{\tau_{\min }}=\exp \left[\frac{2\left(\left\langle i_{c}\right\rangle-i_{0}\right) \sqrt{3 T_{d}}}{T}\right],
$$

We can also write this equation:

$$
i_{0}=\left\langle i_{c}\right\rangle-\frac{\ln \mathcal{R}}{2 \sqrt{3}} \frac{T}{\sqrt{T_{d}}},
$$

which relates the applied current $i_{0}$ to the ratio $T / \sqrt{T_{d}}$ and corresponds to a given dynamical behavior assuming it is set by the value of $\mathcal{R}$. Eq. (6) does not take into account the progressive increase in the local current $i_{\ell}$ resulting from stress intensification as well as the existence of a percolation threshold $i_{p}$ for a given amount of damage $\Phi$ above which a catastrophic rupture of the network will occur spontaneously, i. e. without the help of thermally activated rupture. We find that the various dynamical regimes of rupture can effectively be described by the following equation inspired from eq. (6):

$$
i_{0}=A-C \frac{T}{\sqrt{T_{d}}}
$$


Appropriate values of $A$ and $C$ yield analytical curves separating the three regions in Fig. 5: the solid and dashed line in Fig. 5 correspond to the location of points whose dynamical evolution is really $\operatorname{logarithmic}(p=1)$ and exponential respectively. Note that at the border between these two regions the behaviour is a bit fuzzy, making it more difficult to define a "truly" logarithmic or exponential evolution. We also find that the values obtained for $A$ are close to the typical values of the percolation threshold: for instance, a disorder $T_{d} \sim 0.33$ so that $\min \left(i_{c}\right) \simeq 0$ gives a percolation threshold of the network $i_{p} \simeq 0.236$.

In order to link the rupture dynamics to the spatial distribution of rupture events we show in Figs. 6 and 7 some snapshots of the broken fuses just before the final rupture for different values of $x$ and $i_{0}$. In particular, in Fig. 6 we compare the final snapshots for two rather different values of $x, x=49$ and $x=387$, fixing $i_{0}=0.2$. For large $x$,

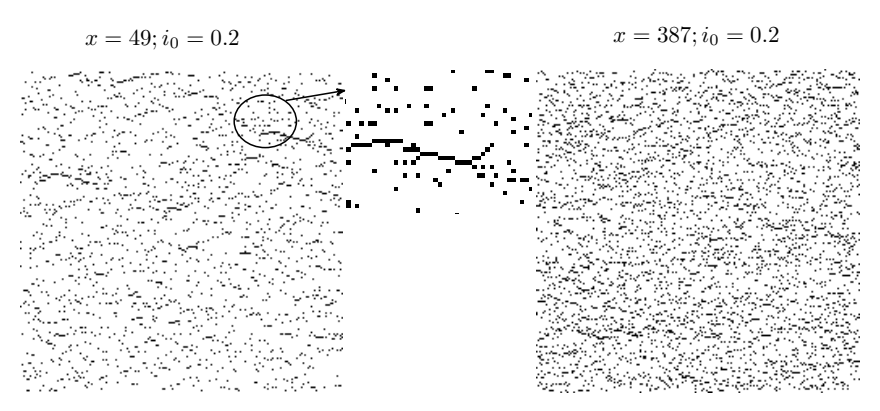

Fig. 6. Snapshot of the broken fuses (black dots) just before the final rupture for $T_{d}=0.001, T=0.02$ and $i_{0}=0.2$ (left), $T_{d}=0.15, T=0.001$ and $i_{0}=0.2$ (right). A zone with rather large cracks is encircled in the left panel and is zoomed in the centre panel.

damage is scattered everywhere in the sample, while for smaller $x$ we notice more localized damage zones and a higher tendency to form straight cracks (in the direction perpendicular to the applied current). Moreover straight cracks tend to coalesce forming larger non-straight cracks (see e.g. the encircled zone in the left panel of Fig. 6 and its zoom in the centre panel). It has to be noted that, especially at low values of $i_{0}$, there is always a background of many diffuse rupture events just before the final rupture of the sample, even at small $x$, although their numbre increases when increasing $x$. In Fig. 7 a comparison of the snapshots of broken fuses for $x=223$ and two extreme values of $i_{0}\left(i_{0}=0.08\right.$ and $\left.i_{0}=0.4\right)$ is shown. The effect of a very large applied current is drastic, since only very few rupture events occur before the final avalanche, while for a very small current the system is more significantly damaged before global failure. This behavior can be understood by considering that for a large applied current $\left(i_{0}>\sqrt{T_{d}}\right)$ it is easier to break a fuse next to an already broken fuse, so that damaging will mainly proceed by growth of cracks and cascade of rupture events.

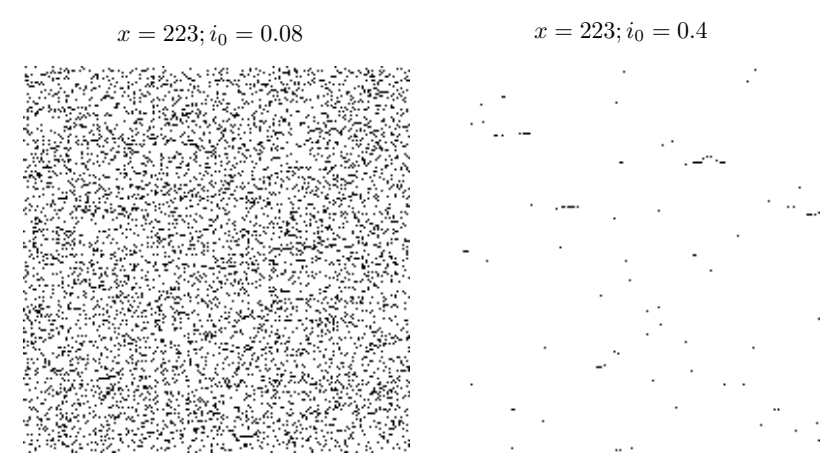

Fig. 7. Snapshot of the broken fuses (black dots) just before the final rupture for $T_{d}=0.2, T=0.002$ and $i_{0}=0.08$ (top), $T_{d}=0.05, T=0.001$ and $i_{0}=0.4$ (bottom).

The same situation will happen when temperature is high enough $(\operatorname{small} x)$. On the other hand, for small applied currents and/or large $x$ such correlations between rupture events will smear out and the resulting damage dynamics will be characterized by a more diffuse distribution of breaking events, which is reflected by a slow logarithmic evolution of the modulus. This is the relevant regime in applications for which materials must have a long lifetime under applied solicitations of moderated amplitudes.

\section{Conclusion}

We have studied the dynamical behaviour of the 2D RFN with disorder and thermal noise, at constant imposed current, by exploring different regions of disorder $T_{d}$, temperature $T$ and applied current $i_{0}$. We have found a rich variety of regimes in the rupture dynamics, ranging from faster than exponential to slower than logarithmic. The transition between the different regimes is mainly determined by the values of $\sqrt{T_{d}} / T$ and $i_{0}$. In particular, for large $\sqrt{T_{d}} / T$ and small $i_{0}$ the modulus displays a (slower than) logarithmic evolution, which is a signature of a slow and diffuse rupture process in the system. This is consistent with recent experimental results where the logarithmic relaxation was connected to the slow rupture dynamics $[42,7]$. Our study suggests that it is therefore important to follow the long-time fracture behaviour and the damage dynamics of the system, which can give an insight on the rupture mechanisms of the material. In our $2 \mathrm{D}$ RFN the material is modeled as a network experiencing scalar elasticity. It would be desirable to extend this model to full $3 \mathrm{D}$ elasticity and to the continuum limit in order to make a closer contact with real experimental systems.

We have modeled the systems with a distribution of rupture threshold. Note that, in terms of activation energy distribution, this is equivalent to a material with a distribution of local intensification of the stress due to heterogeneities of the materials. Such a feature is intrinsic for instance to semi-crystalline polymers for which the 
crystallite lead to local stress amplifications in the amorphous phase, or even more in glass fibers reinforced semicrystalline polymers. Anisotropic rigid inclusions, such as glass fibers, with a length more than ten times larger than the fiber diameters (typically $10 \mu \mathrm{m}$ ) lead to a distribution of local stress spread over more than a decade [6]. Our model allowed us to obtain a phase diagram regarding the dynamical evolution under damaging events of the elastic modulus of disordered materials submitted to an applied stress. This kind of approach should be useful for interpreting slow creep experiments in semi-crystalline polymers, or fatigue experiments in these materials. It should be also of interest for such kind of experiments in a broad class of heterogeneous materials, either with a wide distribution of energy barriers, or with a wide distribution of stress amplification at a microscopic level. Our results have also illustrated that the transition between rapid/exponential damaging and slow/logarithmic damaging is accompanied by a very different rupture pattern: narrow disorder (exponential decay) results in rupture by crack growth, whereas broad disorder results in rupture by accumulation of random nucleation of defects (cavities,...) throughout the sample. This feature will be quantified and studied more systematically in future works. When fully developed, this kind of approach should help for calculating the appropriate level of stress to which parts should be submitted, according to the aimed lifetime of materials, as a function of their disordered nature.

\section{A Technical details of the data analysis}

Starting from Eq. (4), after some algebraic manipulations on the first and second derivative of $E$, we obtain

$$
1-p=\frac{\frac{d^{2} E}{d \log t^{2}}}{\frac{d E}{d \log t}}
$$

$1-p=0,1-p>0$ and $1-p<0$ correspond respectively to a logarithmic, faster than logarithmic and slower than logarithmic decay of $E(t)$. We have determined for each simulation the typical value of $1-p$ computed using eq. 8 and the value of $E$ measured in the numerical simulation. As the rupture process is statistical, an average over several simulations in the same conditions has been done to obtain $E(t)$ [40]. However, the resulting first and second derivatives computed in eq. 8 will still be rather noisy. In order to take into account this noise, we use the following procedure. First, we introduce the time $t^{*}$ at which the modulus has halved with respect to its value $E_{\text {min }}$ reached at the final breaking stage:

$$
E\left(t^{*}\right)=\frac{1}{2}\left(E_{0}+E_{\text {min }}\right) .
$$

This choice of $t^{*}$ has been made to avoid the time boundaries and in particular the initial flat region of $E(t)$. We look for the time interval $\left(t_{\min }, t_{\max }\right)$ in which $1-p$ is a constant within a tolerance $\Delta_{\text {const }}$ :

$$
\left|1-p(t)-\left(1-p\left(t^{*}\right)\right)\right|<\Delta_{\text {const }} .
$$

We have chosen $\Delta_{\text {const }}=0.4$. Then we calculate the average value of $1-p$ in $\left(t_{\min }, t_{\max }\right)$ and we look at its sign to determine the type of evolution of $E(t)$, i.e. logarithmic, or faster (slower) than logarithmic.

Besides the analysis of the logarithmic evolution, we have also performed exponential fits to the initial time region of $E(t)$ in order to assess the occurrence of a faster or slower than exponential decay of the modulus.

As already mentioned in [40], we stress here that all the quantities analysed, such as $E, \frac{d E}{d t}, \frac{d^{2} E}{d t^{2}}$ and $1-p$, are averaged over 10 realizations. We have also introduced an extra filter in the data analysis considering only cases where lifetime is larger than 1000 .

\section{References}

1. Statistical Models for the Fracture of Disordered Media, edited by S. Roux and H. J. Herrmann (North-Holland, Amsterdam, 1990).

2. B. K. Chakrabarti and L. G. Benguigui, Statistical Physics of Fracture and Breakdown in Disordered Systems (Clarendon Press, Oxford, 1997).

3. M. J.Alava, P. K. V. V. Nukala and S. Zapperi, Statistical models of fracture, Adv. Phys. 55, 349 (2006).

4. L. Vanel, S. Ciliberto, P.-P. Cortet, and S. Santucci, Timedependent rupture and slow crack growth: elastic and viscoplastic dynamics, J. Phys. D: Appl. Phys. 42(21), 214007 (2009).

5. R.W. Hertzberg and John A. Manson, Fatigue of Engineering Plastics (Academic Press, New York, 1980)

6. E. Mourglia, PhD Thesis, Laboratoire de Polymères et Matériaux Avancés, CNRS/Rhodia, Lyon, France (2010).

7. E. Mourglia, D. Long, L. Odoni, P. Sotta and C. Rochas, Physical mechanisms during fatigue testing of reinforceystalline polymers, Proceedings of the $14^{\text {th }}$ International Conference on Deformation, Yield and Fracture of Polymers, pag. 321 (2009).

8. Mallick, P. K.; Zhou, Y. Effect of mean stress on the stress-controlled fatigue of a short E-glass fiber reinforced polyamide-6,6. International Journal of Fatigue (2004), 26(9), 941-946.

9. Horst, J. J.; Spoormaker, J. Mechanisms of Fatigue in Short Glass Fiber Reinforced Polyamide 6. Polymer Engineering and Science (1996), 36 (22), 2718-2726.

10. Zhou, Y.; Mallick, P. K. Fatigue Performance of an Injection-Molded Short E-Glass Fiber-Reinforced Polyamide 6,6. I. Effects of Orientation, Holes, and Weld Line. Polymer Composites (2006), 27 (2), 230-237.

11. Bellenger, V.; Tcharkhtchi, A.; Castaing, P. Thermal and mechanical fatigue of a PA66/glass fibers composite material. International Journal of Fatigue (2006), 28 (10), 1348-1352.

12. Bernasconi, A.; Kulin, M. Effect of Frequency Upon Fatigue Strength of a Short Glass Fiber Reinforced Polyamide 6: A Superposition Method Based on Cyclic Creep Parameters. Polymer Composites (2009), 30 (2), 154-161.

13. Dally, J. W. Fatigue Behavior of Glass-Fiber Fortified Thermoplastics. Polymer Engineering and Science (1969), 9 (6), 434-444. 
14. Castagnet, S.; Girault, S.; Gacougnolle, J. L.; Dang, P. Cavitation in strained polyvinylidene fluoride: mechanical and X-ray experimental studies. Polymer (2000), 41, 75237530.

15. Pawlak, A.; Galeski, A. Plastic Deformation of Crystalline Polymers: The Role of Cavitation and Crystal Plasticity. Macromolecules (2005), 38, 9688-9697.

16. Mandell, J. F.; Huang, D. D.; McGarry, F. J. Fatigue of Glass and Carbon Fiber Reinforced Engineering Thermoplastics. Polymer Composites (1981), 2 (3), 137-144.

17. Lesser, A. J. Changes in Mechanical behavior During Fatigue of Semicrystalline Thermoplastics. Journal of Applied Polymer Science (1995), 58, 869-879.

18. Bowden, P. B.; Jukes, J. A. The Plastic Flow of Isotropic Polymers. Journal of Materials Science (1972), 7, 52-63.

19. V.S. Kuksenko and V.P. Tamuzs, Fracture micromechanics of polymer materials (Martinus Nijhoff Publishers, The Hague, 1981).

20. B. Kahng, G. G. Batrouni, S. Redner, L. De Arcangelis and H. J. Herrmann, Phys. Rev. B 37, 7625 (1988).

21. S. Zapperi, P. Ray, H. E. Stanley and A. Vespignani, Phys. Rev. E 59, 5049 (1999).

22. J. V. Andersen, D. Sornette and K.-T. Leung, Phys. Rev. Lett. 78, 2140 (1997).

23. P.-P. Cortet, L. Vanel and S. Ciliberto, Europhys. Lett. 74, 602 (2006).

24. S. Santucci, L. Vanel, A. Guarino, R. Scorretti and S. Ciliberto, Europhys. Lett. 62, 320 (2004).

25. R. Toussaint and A. Hansen, Phys. Rev. E 73, 046103 (2006).

26. D. De Tommasi, S. Marzano, G. Puglisi and G. Saccomandi, Continuum Mech. Thermodyn. 22, 47 (2010).

27. A. Garciamartin, A. Guarino, L. Bellon and S. Ciliberto, Phys. Rev. Lett. 79, 3202 (1997).

28. E. Bouchaud, J. Phys.: Condens. Matter 9, 4319 (1997).

29. D. Bonamy, S. Santucci and L. Ponson, Phys. Rev. Lett. 101, 045501 (2008).

30. D. Bonamy, J. Phys. D 42, 214014 (2009).

31. F. Reurings and M. J. Alava, Eur. Phys. J. B 47, 85 (2005).

32. S. Roux, Phys. Rev. E 62, 6164 (2000).

33. S. Ciliberto, A. Guarino and R. Scorretti, Physica D 158, 83 (2001).

34. R. Scorretti, S. Ciliberto and A. Guarino, Europhys. Lett. 55, 626 (2001)

35. A. Politi, S. Ciliberto and R. Scorretti, Phys. Rev. E 66, 026107 (2002).

36. A. Saichev and D. Sornette, Phys. Rev. E 71, 016608 (2005).

37. A. Guarino, L. Vanel, R. Scorretti and S. Ciliberto, J. Stat. Mech., P06020 (2006).

38. A. Guarino and S. Ciliberto, S. Eur. Phys. J. B 83(2), 215 (2011).

39. N. Shahidzadeh-Bonn, P. Vi, X. Chateau, J.-N. Roux and D. Bonn, Phys. Rev. Lett. 95, 175501 (2005).

40. We have performed numerical simulations of the model for different values of the parameters $T_{d}, T$ and $i_{0}$ and for fixed system size $M \times N=200 \times 200$. The time scale in the simulations is determined by the thermal noise, i.e. at each time step we extract $\delta i_{\ell}$ according to the Gaussian distribution. Since the disorder is quenched, we fix the threshold distribution at the beginning of the simulation. In order to reduce statistical fluctuations we average the results over 10 different realizations for the same set of parameters.
41. T. Utsu, Geophys. Mag. 30, 521 (1995).

42. N. Mallick, S. Ciliberto, S. G. Roux, P. Di Stefano and L. Vanel, Aftershocks in thermally activated rupture of indented glass, Proceedings of the $12^{\text {th }}$ International Conference on Fracture, 12-17 juillet 2009, Ottawa, communication T48.003. 